\title{
Multiplexed VaxArray immunoassay for rapid antigen quantification in measles and rubella vaccine manufacturing
}

\author{
Jacob H. Gillis ${ }^{\mathrm{a}, \mathrm{c}}$, Keely N. Thomas ${ }^{\mathrm{a}}$, Senthilkumar Manoharan ${ }^{\mathrm{b}}$, Mallikarjuna Panchakshari ${ }^{\mathrm{b}}$, \\ Amber W. Taylor ${ }^{a}$, David F. Miller ${ }^{a}$, Rose T. Byrne-Nash ${ }^{\mathrm{a}, \mathrm{c}}$, Christine Riley ${ }^{\mathrm{a}}$, Kathy L. Rowlen ${ }^{\mathrm{a}}$, Erica \\ Dawson $^{\mathrm{a}, *}$
}

a InDevR Inc., Boulder, CO, USA

${ }^{\mathrm{b}}$ Biological E. Ltd., Hyderabad, India

${ }^{\mathrm{c}}$ GT Molecular, Fort Collins, CO, USA

\section{A R T I C L E I N F O}

\section{Article history:}

Received 23 March 2021

Received in revised form 12 July 2021

Accepted 1 September 2021

Available online 20 September 2021

\section{Keywords:}

Measles

Rubella

$\mathrm{CCID}_{50}$

Identification

Antigen Quantification

MR Vaccine

Vaccine Manufacturing

Multiplexed Immunoassay

Microarray

VaxArray

\begin{abstract}
A B S T R A C T
Measles-containing vaccines (MCV), specifically vaccines against measles and rubella (MR), are extremely effective and critical for the eradication of measles and rubella diseases. In developed countries, vaccination rates are high and vaccines are readily available, but continued high prevalence of both diseases in developing countries and surges in measles deaths in recent years have highlighted the need to expand vaccination efforts. To meet demand for additional vaccines at a globally affordable price, it is highly desirable to streamline vaccine production thereby reducing cost and speeding up time to delivery. MR vaccine characterization currently relies on the $50 \%$ cell culture infectious dose $\left(C C_{50}\right)$ assay, an endpoint assay with low reproducibility that requires 10-14 days to complete. For streamlining bioprocess analysis and improving measurement precision relative to $\mathrm{CCID}_{50}$, we developed the VaxArray Measles and Rubella assay kit, which is based on a multiplexed microarray immunoassay with a 5hour time to result. Here we demonstrate vaccine-relevant sensitivity ranging from 345 to $800 \mathrm{IFU} / \mathrm{mL}$ up to $100,000 \mathrm{IFU} / \mathrm{mL}$ (infectious units per $\mathrm{mL}$ ) and specificity that allows simultaneous analysis in bivalent vaccine samples. The assay is sensitive to antigen stability and has minimal interference from common vaccine additives. The assay exhibits high reproducibility and repeatability, with $15 \% \mathrm{CV}$, much lower than the typical $0.3 \log _{10}$ error ( 65\%) observed for the $\mathrm{CCID}_{50}$ assay. The intact protein concentration measured by VaxArray is reasonably correlated to, but not equivalent to, $\mathrm{CCID}_{50}$ infectivity measurements for harvest samples. However, the measured protein concentration exhibits equivalency to $\mathrm{CCID}_{50}$ for more purified samples, including concentrated virus pools and monovalent bulks, making the assay a useful new tool for same-day analysis of vaccine samples for bioprocess development, optimization, and monitoring.
\end{abstract}

(C) 2021 The Author(s). Published by Elsevier Ltd. This is an open access article under the CC BY license (http://creativecommons.org/licenses/by/4.0/).

\section{Introduction}

Measles and rubella are highly contagious viruses that cause significant morbidity and mortality. Measles virus is one of the most infectious human-borne viruses and, prior to widespread vaccination, infected $>90 \%$ of children before 15 years of age [1]. Acute measles infection is associated with fever, cough, and rash that can persist for a week [2]. Up to $6 \%$ of acute measles cases are fatal and the World Health Organization (WHO) estimates 140,000 children, most in the developing world, died from acute measles infection in 2018 [3]. Subacute sclerosing panencephalitis (SSPE), a chronic

\footnotetext{
* Corresponding author.

E-mail address: dawson@indevr.com (E. Dawson), dawson@indevr.com (E.
}

complication of measles infection, can occur in patients up to 10 years after initial acute infection. While rare $(0.007-0.011 \%$ of infections [4]), SSPE is always fatal, usually within 1 to 3 years.

Unlike measles, acute rubella infection is rarely fatal. Rubella infection during the first trimester of pregnancy is associated with severe chronic developmental disorders (congenital rubella syndrome, CRS) in the developing fetus that includes cataracts, deafness, encephalopathy, heart defects, and severe mental development disorders. When infected during the first 12 weeks of gestation, up to $80 \%$ of fetuses will develop CRS [5,6]. Prior to introduction of rubella vaccination (and in non-immunized populations), CRS occurs at a rate of $0.8-4.0$ per 1000 live births [7], though this likely greatly under-reports the occurrence [8]. 
Current measles vaccines are up to $99 \%$ effective, but high coverage rates of $95 \%$ or greater are necessary for elimination of measles [9-11]. Rubella vaccines have demonstrated similar efficacy $[8,12,13]$, reducing the incidence of CRS in newborns to $<20$ total per year in WHO regions with high $(>90 \%)$ vaccination coverage [14].

Measles-containing vaccines (MCV) are affordable, available, and have been in use for a half-century in the developed world, but coverage in low- and middle-income countries is lacking due in part to cold-chain requirements and manufacturing costs. Several initiatives aim to enable low-cost manufacturing of measles and rubella (MR) vaccines at the quantity and cost required for widespread vaccination in the developing world [15]. Recent efforts have been highly successful, reducing the number of measles-associated deaths by $74 \%$ worldwide between 2000 and 2010 [16], but a 50\% increase in measles mortality worldwide between 2016 and 2019 [16] highlights the fight is not over.

MR vaccines rely on cell-culture based assays, such as 50\% Cell Culture Infectious Dose $\left(\mathrm{CCID}_{50}\right)$, for characterization during manufacturing, including identity, potency, and stability testing. Infectivity measurements are widely adopted and highly predictive of vaccine efficacy [17-18] but present certain limitations, as the time to result is 10-14 days and execution requires trained personnel. In addition, $\mathrm{CCID}_{50}$ assays rely on subjective, discontinuous endpoint measurements and are notoriously error prone with observed variability of $\pm 0.3 \log _{10}$ infectious units per $\mathrm{mL}$ (50-100\% variability) [19-20], resulting in costly hold times and lot rejections that can increase manufacturing costs and delay delivery of critical vaccine doses.

This work describes the development of an alternative assay for tracking antigen content, stability, and identity throughout the MR vaccine manufacturing process. We have adapted the VaxArray technology, described previously for influenza vaccines [21-24], for the evaluation of MR vaccine antigens. VaxArray assays are simple multiplexed immunoassays that use monoclonal antibodies in a glass microarray format. In addition to multiplexing, VaxArray provides a significant advantage in terms of reagent use, requiring 100x less capture antibody than ELISA. Here we demonstrate feasibility of the VaxArray technology for measuring antigen content in MR vaccines, enabling streamlined characterization at several manufacturing steps.

\section{Materials and Methods}

\subsection{Anti-measles and rubella antibodies}

Antibodies raised against measles and rubella virus-specific were obtained from numerous commercial sources, including Lifespan Bioscience (Seattle, WA), Novus Biologicals (Littleton, CO), Fitzgerald Industries International (Acton, MA), GeneTex (Irvine, CA), ViroStat Inc. (Westbrook, ME), Antibodies-Online (Aachen, Germany), MilliporeSigma (Burlington, MA), and HyTest Ltd (Turku, Finland).

\subsection{VaxArray MR assay}

The VaxArray Measles and Rubella (MR) assay is similar to previously-described VaxArray Influenza assays [21-24], with the slide layout, microarray layout, and detection principle depicted in Fig. 1a, b, and c, respectively. A single virus-specific monoclonal antibody against each virus is printed on the microarray (Fig. 1A) and used to capture viral antigens which are detected via a fluorescent antibody. Each VaxArray MR assay kit (VXMR-9001, InDevR Inc.) contains two microarray slides, each with 16 replicate arrays (see Fig. 1a and 1b), MR Blocking Buffer (MRBB), MR Lysis Buffer (4x), and Wash Buffers. Prior to use, VaxArray MR slides were equilibrated at $25{ }^{\circ} \mathrm{C}$ for $30 \mathrm{~min}$. Samples were prepared individually at $3 \mathrm{x}$ final concentration by lysing at $25^{\circ} \mathrm{C}$ for $30 \mathrm{~min}$ in $1 \mathrm{x}$ PBS $+1 \mathrm{x}$ MR Lysis Buffer. Each sample was further diluted in MR Blocking Buffer to $1 \mathrm{x}$, and $50 \mu \mathrm{L}$ was applied to individual arrays. Slide(s) were incubated in a humidity chamber (VX-6200, InDevR Inc.) on an orbital shaker at $65 \mathrm{rpm}$ for 4 to $20 \mathrm{~h}$ at $25^{\circ} \mathrm{C}$. MR Blocking Buffer and antigen-specific detection label (VXMR-7634 and VXMR7635 , InDevR Inc.) was prepared and added to each array following antigen removal. Each slide was further incubated for $30 \mathrm{~min}$ in the humidity chamber at $65 \mathrm{rpm}$ at $25^{\circ} \mathrm{C}$ before subsequent, sequential washes with Wash Buffer 1, Wash Buffer 2, 70\% ethanol, and water. Slides were dried and imaged using the VaxArray Imaging System (VX-6000, InDevR Inc.). Fluorescence intensities were processed using the validated VaxArray Analysis Software utilizing the algorithms of Kuck et al. 24]. When appropriate, sample concentrations were calculated against a standard curve from the same experiment.

\subsection{Cell culture}

Growth medium for Vero cells (CCL-81, ATCC) consisted of Medium 199 (11150059, Gibco) supplemented to 5\% fetal bovine serum (A3160401, Gibco) plus 2 mM L-Glutamine (A2916801, Gibco) and 1x Penicillin-Streptomycin (15140148, Gibco). Diluent medium for Vero cells consisted of Medium 199 (11150059, Gibco) supplemented to $2 \%$ fetal bovine serum (A3160401, Gibco) plus $2 \mathrm{mM}$ L-Glutamine (A2916801, Gibco) and 1x PenicillinStreptomycin (15140148, Gibco).

Vero cells were grown in Vero growth media in adherent cell culture flasks (Nunc EasYFlask) to 80-90\% confluency. Cells were collected by washing adherent cells with sterile Dulbecco's PBS (14190136, Gibco) followed by incubation for $\sim 5 \mathrm{~min}$ with $0.25 \%$ Trypsin-EDTA (25200056, ThermoFisher). Cells were then collected, and trypsin was inactivated with an equivalent volume of supplemented growth medium.

Growth medium for Rabbit Kidney (RK13) cells (CCL-37, ATCC) consisted of Dulbecco's Minimum Essential Medium (12-614F, Lonza) supplemented to $10 \%$ fetal bovine serum (A3160401, Gibco) plus $2 \mathrm{mM}$ L-Glutamine (A2916801, Gibco) and 1x PenicillinStreptomycin (15140148, Gibco). Diluent medium for RK13 cells consisted of Dulbecco's Minimum Essential Medium (12-614F, Lonza) supplemented to $5 \%$ fetal bovine serum (A3160401, Gibco) plus $2 \mathrm{mM}$ L-Glutamine (A2916801, Gibco) and 1x PenicillinStreptomycin (15140148, Gibco).

Cell cultures RK13 cells were grown in RK13 growth media in adherent cell culture flasks (Nunc EasYFlask) to 80-90\% confluency. Cells were collected by washing adherent cells with sterile Dulbecco's PBS (14190136, Gibco) followed by incubation for $\sim 5$ min with $0.25 \%$ Trypsin-EDTA (25200056, ThermoFisher). Cells were then collected, and trypsin was inactivated with an equivalent volume of supplemented growth medium.

\subsection{Cell culture infectious dose $\left(C C I D_{50}\right)$}

Cell Culture Infectious Dose $\left(\mathrm{CCID}_{50}\right)$ assays were performed at InDevR Inc. and Biological E. Ltd. following Biological E. Ltd. protocols. Virus-containing samples were initially diluted in cell-line matched diluent medium and further serially diluted by $0.5 \log _{10}$ in diluent medium to create 12 virus dilutions. $50 \mu \mathrm{L}$ diluent medium was added to all wells of treated 96-well plate(s) (161093, Nunc), and $50 \mu \mathrm{L}$ serially diluted sample were added to each row of the plate ( 8 replicates each dilution). Control plates (no virus) were generated by adding an extra $50 \mu \mathrm{L}$ diluent medium.

Vero cells were utilized for measles virus, and RK13 cells for rubella virus. Cell cultures were started $\sim 1$ week prior to execut- 


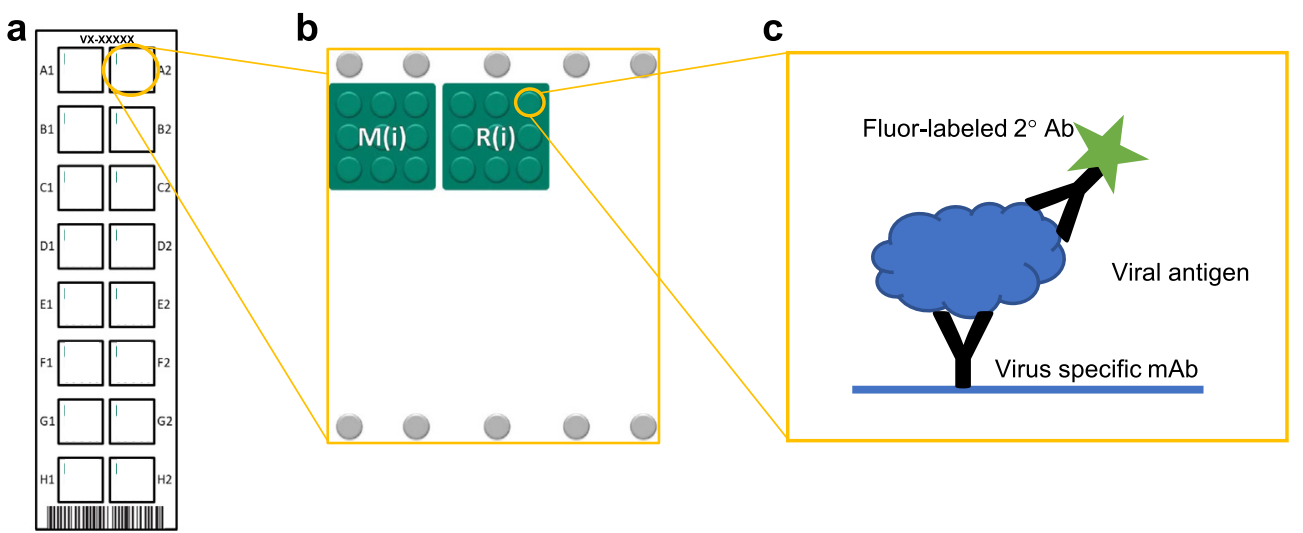

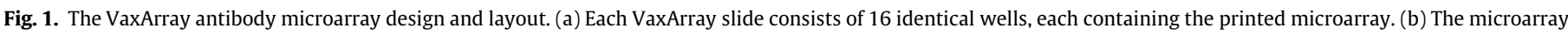

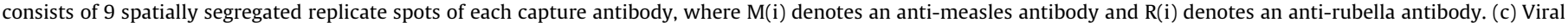

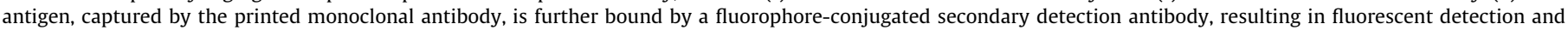
quantification.

ing $\mathrm{CCID}_{50}$. Cells were grown in a $\mathrm{CO}_{2}$ incubator at $37{ }^{\circ} \mathrm{C}$ and $5 \%$ $\mathrm{CO}_{2}$ to $80-90 \%$ confluency in T175 flasks, collected by trypsin release as described above, and cell concentration determined via hemocytometer. Cells were collected, centrifuged, and reconstituted in diluent medium to create $13 \mathrm{~mL}$ of $1.1 \times 10^{5}$ cells $/ \mathrm{mL}$ solution per $\mathrm{CCID}_{50}$ plate. $100 \mu \mathrm{L}$ cell solution was added to every well of every plate, including the control plate.

Plate(s) were covered and placed in $\mathrm{CO}_{2}$ incubators at $32{ }^{\circ} \mathrm{C}$ (rubella) or $36{ }^{\circ} \mathrm{C}$ (measles). Plates were incubated for 10 days with periodic inspection under an inverted microscope. At the end of the 10-day incubation, all wells of the plate(s) were inspected for cytopathic effects. A Spearman-Karber calculation $[25,26]$ was applied to determine the $\mathrm{CCID}_{50}$ titer of each sample.

\subsection{Virus-containing samples}

Three (3) lots of monovalent bulk containing live-attenuated measles CAM-70 strain, 4 lots of monovalent bulk containing live-attenuated rubella Wistar RA 27/3 strain, 3 lots of lyophilized vaccine containing measles CAM-70 and rubella Wistar RA 27/3, and 17 rubella Wistar RA 27/3-containing samples, from 2 separate lots (3 different growth conditions for one lot, and a single growth condition for the second lot), harvested at different infection times ('harvest samples'), were obtained from Biological E. Ltd. (Hyderabad, India). A separate set of 12 harvest samples containing measles Schwarz from a single lot of material but representing 3 different growth conditions were provided by Batavia Biosciences B.V. (Leiden, Netherlands).

\subsection{Analytical sensitivity and linear dynamic range}

Samples were lysed using MR Lysis Buffer (VXMR-6310, InDevR) for $30 \mathrm{~min}$ at $25^{\circ} \mathrm{C}$ in a biosafety cabinet. Samples were serially diluted in MR Blocking Buffer (VXMR-6309, InDevR) to create a 13-point standard curve. Test samples at low virus concentrations were lysed and diluted in MR Blocking Buffer prior to analysis $(\mathrm{n}=4) .50 \mu \mathrm{L}$ of each standard was added to individual arrays on VaxArray Measles and Rubella v1.0 assay slides (VXMR-9051, InDevR), and further processed using virus-specific detection labels with slides imaged at $700 \mathrm{~ms}$ exposure time.

A linear regression was applied to each set of 4 adjacent standards across the 13-pt dataset with each slope and $\mathrm{R}^{2}$ calculated. The upper limit of quantification (ULOQ) was defined as the highest antigen concentration analyzed that was within a 4-point fit $\mathrm{R}^{2}>0.95$. To determine the lower limit of quantification (LLOQ), the low-concentration test samples were quantified against the matched standard curve and the average concentration and standard deviation across 4 replicates determined. LLOQ was defined as the lowest concentration with a \%CV $<25 \%$ and a \% difference from expected (accuracy) $<25 \%$.

\subsection{Analytical specificity and accuracy in bivalent samples}

Monovalent bulk measles CAM-70 and rubella RA 27/3 samples and bivalent mixtures of the two were prepared by serially diluting in MR Blocking Buffer, with monovalent and corresponding bivalent samples containing the same concentrations. Samples were analyzed by the VaxArray Measles and Rubella Assay as described previously. To determine specificity and accuracy of bivalent analysis, the assay response for a virus analyzed in monovalent form was compared to the same response when analyzed in the bivalent sample and the statistical difference between the two regressions compared.

\subsection{Assay precision}

On three days, three separate users executed the VaxArray MR assay. On each day, a bivalent mixture of measles Schwarz (harvest sample) and rubella RA 27/3 (monovalent bulk) was prepared in PBS. The highest standard was diluted in PBS, lysed, and further diluted in MR Blocking Buffer to create the standards. In addition, 3 samples (high, middle, and low concentrations) were generated in PBS, with each lysed 8 separate times before dilution in MR Blocking Buffer. $50 \mu \mathrm{L}$ of each standard and sample replicate were added to individual microarrays and processed as described previously. The average sample concentration and standard deviation for each sample were determined $(n=8)$. Day-to-day results were compared for each sample, with results also normalized by their dilution factor to compare all replicates $(n=72)$.

\subsection{Correlation and accuracy relative to $C C I D_{50}$}

Monovalent harvest samples (measles Schwarz or rubella RA $27 / 3$ ) were prepared and analyzed by both VaxArray $\mathrm{CCID}_{50}$ assay previously described. Seventeen (17) rubella harvest samples were analyzed, using a $\mathrm{CCID}_{50}$-characterized monovalent bulk as the VaxArray calibrant. Eleven (11) measles harvest samples were analyzed. As the difference in titer for the 11 samples was small, each sample was also further diluted twice to create 33 total samples. Measles samples were then analyzed by VaxArray and $\mathrm{CCID}_{50}$ 
using a separate harvest sample previously measured by $\mathrm{CCID}_{50}$ as the VaxArray calibrant.

Separately, 5 concentrated virus pools (CVP), 3 monovalent rubella RA 27/3 bulks, and 2 monovalent measles CAM-70 bulks, all from separate manufacturing lots were analyzed by VaxArray MR and CCID $_{50}$. Additionally, 3 final vaccines (measles CAM-70 and rubella RA27/3) from separate lots were analyzed. A separate monovalent bulk for each virus from a different lot with known $\mathrm{CCID}_{50}$ was used as the VaxArray calibrant.

\subsection{Thermal degradation/stability indication}

CAM-70 measles and RA 27/3 rubella monovalent bulk samples were aliquoted into separate glass vials, sealed with a rubber stopper and aluminum crimp-top, and frozen at $-80{ }^{\circ} \mathrm{C}$. 'Treated' vials were then placed in a water bath at $+60{ }^{\circ} \mathrm{C}$ for $48 \mathrm{~h}$ or in an incubator at $+37^{\circ} \mathrm{C}$ for $24 \mathrm{~h}$, after which the 'untreated' vials were removed from the freezer and allowed to equilibrate to $25^{\circ} \mathrm{C}$ in a biosafety cabinet. Untreated and thermally treated materials were combined in specific ratios, and each prepared sample was lysed and analyzed by VaxArray. The assay response for each tested sample was compared to the fully intact sample and a \% signal was determined. The percent signal relative to intact material was plotted against the \% intact material and a linear regression was applied.

\subsection{Interfering substances}

Separately, solutions containing 5\% sucrose (S9378, Sigma), $1.125 \%$ sodium chloride (S3014, Sigma), 6.25\% sorbitol (S1876, Sigma), 1\% Tween 80 (BP338, Fisher Scientific), and 2\% gelatin (G1393, Sigma) were prepared (w/v) in deionized water. A bivalent mixture of measles and rubella virus was prepared and combined with each vaccine-relevant matrix, resulting in samples of virus plus $0.38 \%$ sucrose, $0.90 \%$ sodium chloride, $5 \%$ sorbitol, $0.125 \%$ Tween 80 , or $1.60 \%$ gelatin. A control was prepared by diluting in PBS (P3813, Sigma). Each sample was lysed using MR Lysis Buffer for 30 min, further diluted in MR Blocking Buffer to 6x LLOQ with each added to 4 replicate arrays. A standard curve was prepared by serially diluting the PBS-control sample in MR Blocking Buffer after lysis. All slides were processed and imaged as described previously, and samples were quantified against the relevant standard curve and the average concentration of each virus in each sample determined. A student's T-test evaluated the statistical significance of each sample relative to the control.

\section{Results and discussion}

\subsection{Antibody pair selection}

Antibodies against measles nucleoprotein and rubella E1, E2, and capsid proteins were obtained and printed on a "screening" microarray. A panel of 21 monoclonal antibodies, raised against measles $(n=9)$ or rubella $(n=12)$ were each printed in triplicate on the microarray Antibodies were evaluated for reactivity, specificity, limit of detection, linear dynamic range, and stability indicating capabilities, with a single antibody for each virus was down-selected for inclusion in the final assay. The measles antibody targets the nucleoprotein (NP), the most abundant protein in the virus particle and to which antibodies are produced most rapidly and in high numbers [27]. The rubella antibody chosen targets the E1 surface glycoprotein, a major immunogen [28]. The exact binding epitopes of the antibodies chosen are not known.
3.2. VaxArray MR assay has broad linear dynamic ranges and vaccinerelevant limits of quantification

To assess assay linearity, monovalent measles- and rubellacontaining samples were analyzed to determine the ULOQ and LLOQ of the assay for each strain analyzed. As shown in Fig. 2, the assay demonstrated good sensitivity and linearity for CAM-70 measles (Fig. 2.a), Schwarz measles (Fig. 2.b) and Wistar RA 27/3 rubella (Fig. 2.c). Fig. 2.c inset further highlights the linear response to lower-concentration dilutions of the rubella sample. Each strain showed linearity over $\geq 43 \mathrm{x}$ and resulted in LLOQ from 800 to 345 infectious units per $\mathrm{mL}$, or IFU/mL (Table 1). Importantly, these LLOQ values are < 2,000 IFU/mL (3.3 $\left.\log _{10} \mathrm{IFU} / \mathrm{mL}\right)$ minimum required in the final vaccine [18] and are sufficient for antigen tracking throughout vaccine manufacturing.

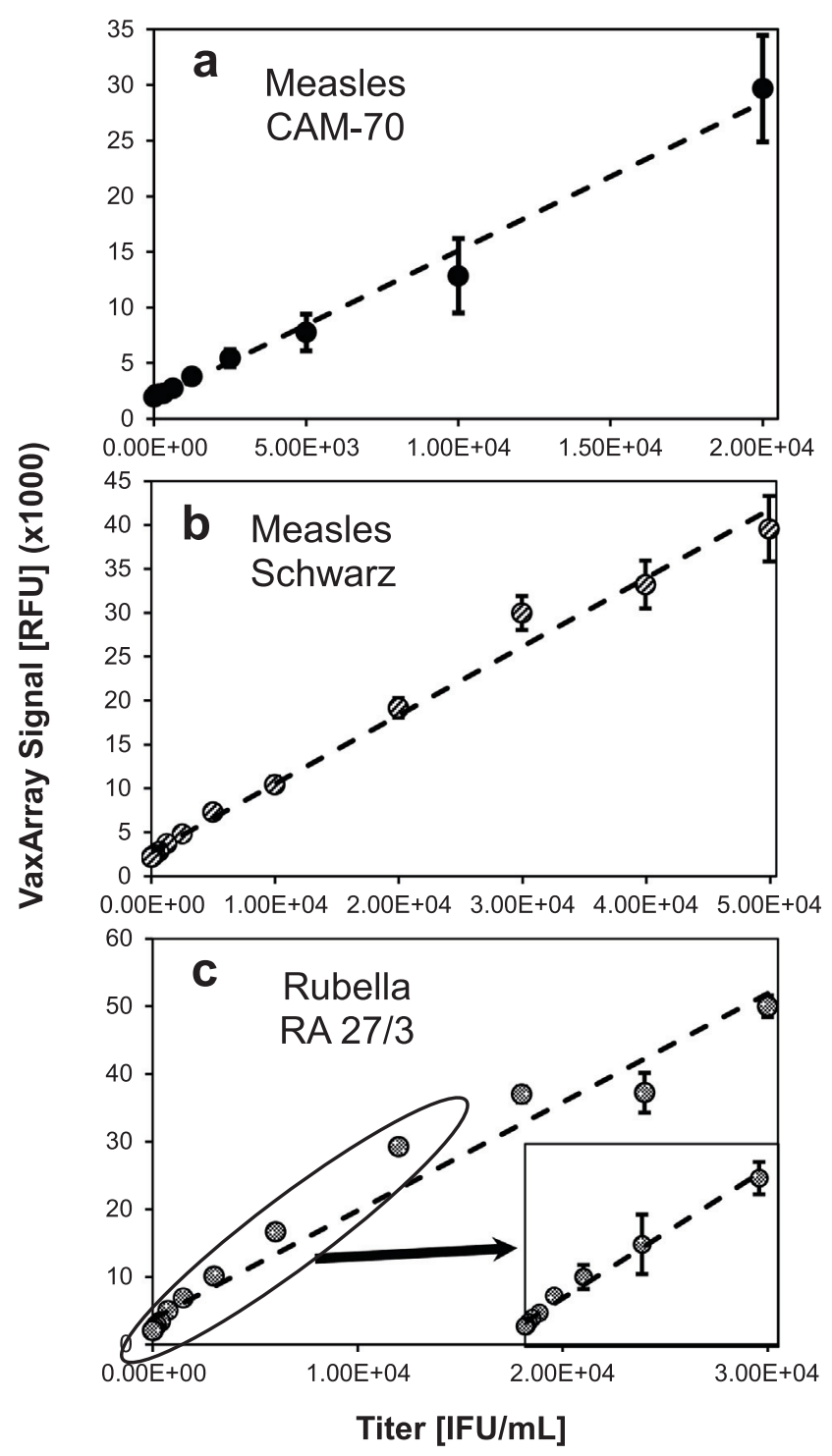

Fig. 2. VaxArray MR assay response curves for serial dilutions of (a) measles CAM70 strain, (b) measles Schwarz strain, and (c) rubella Wistar RA 27/3 strain. Values shown are median signal intensities, and error bars represent \pm 1 standard deviation of the 9 replicate spots within the array for the relevant antibody. 
Table 1

Limits of Quantification of the VaxArray Measles and Rubella Assay.

\begin{tabular}{|c|c|c|c|c|}
\hline Virus & Strain & Lower Limit of Quantification [IFU/mL] & Upper Limit of Quantification [IFU/mL] & Linear Dynamic Range \\
\hline \multirow[t]{2}{*}{ Measles } & CAM-70 & 800 & 100,000 & $125 x$ \\
\hline & Schwarz & 345 & 50,000 & $145 x$ \\
\hline Rubella & Wistar RA $27 / 3$ & 360 & 15,500 & $43 x$ \\
\hline
\end{tabular}

3.3. VaxArray MR assay is capable of accurate, simultaneous bivalent measles and rubella analysis

To investigate the suitability of the VaxArray MR assay for bivalent analysis, measles and rubella monovalent bulk stocks were prepared and serially diluted in monovalent form as well as combined and serially diluted as a bivalent sample (same virus concentrations present in the bivalent and corresponding monovalent samples). Each sample was analyzed and the signal responses compared to determine if the presence of the off-target virus affected accurate quantification of the target virus.

As shown in Fig. 3a, when a measles-only sample is analyzed, only the measles-specific capture antibody shows a response while the signal intensity of the rubella-specific antibody is $<1.02 \mathrm{x}$ background. Similarly, as shown in Fig. $3 \mathbf{b}$, a rubella-only sample demonstrates detection only on the rubella-specific capture antibody, with the signal on the measles-specific antibody is $1.16 \mathrm{x}$ background. A representative image of a bivalent analysis is shown in Fig. 3c. Direct comparison of response curves for equivalent virus concentrations in monovalent and bivalent formulations are shown in Fig. $3 \mathbf{d}$ and Fig. 3e. For measles, the linear regressions through the monovalent and bivalent data series shown in Fig. 3d have similar slopes and y-intercepts. Similarly, the rubella-specific antibody demonstrated minimal difference in assay response in monovalent vs. bivalent samples (Fig. 3e). These results indicate that the analysis of one virus is not inhibited by and does not interfere with the presence of the other virus. The ability to simultaneously quantify both components in bivalent samples provides a distinct advantage, as the VaxArray MR assay does not require the anti-rubella neutralizing serum required for the $\mathrm{CCID}_{50}$ assay of measles due to the concomitant growth of rubella in Vero cells.

\subsection{VaxArray MR assay is compatible with common vaccine additives}

It is also important to investigate potential interference from common stabilizers and excipients typically added to MR vaccine samples to improve shelf stability and assist in the freeze-drying process. To investigate potential interferents, a bivalent mixture of measles and rubella bulks was spiked into five matrices at vaccine-relevant concentrations[29]. a

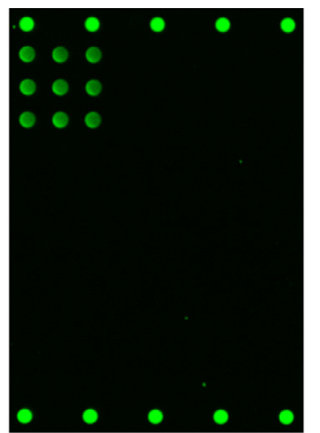

d

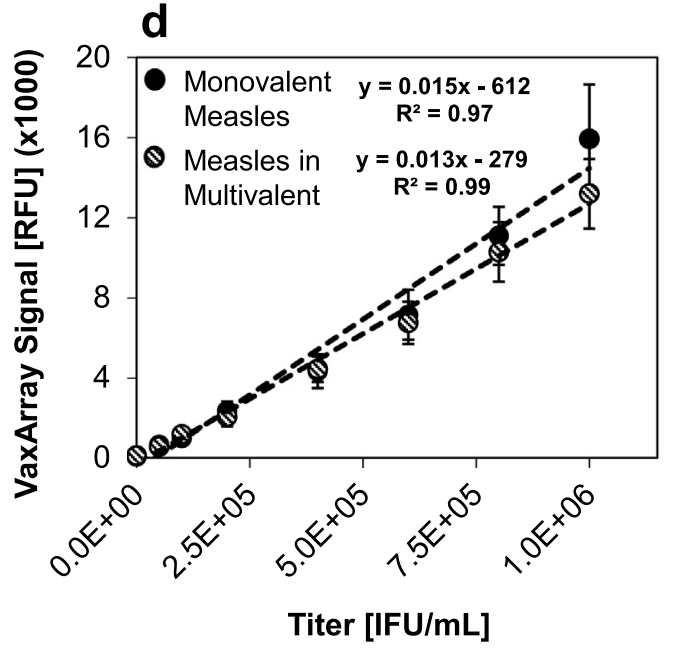

b

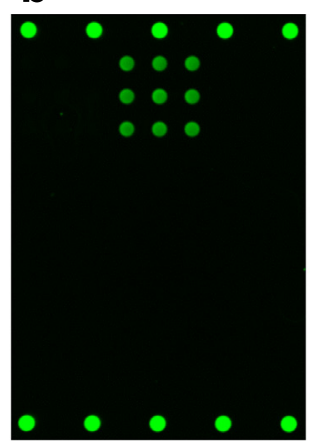

C

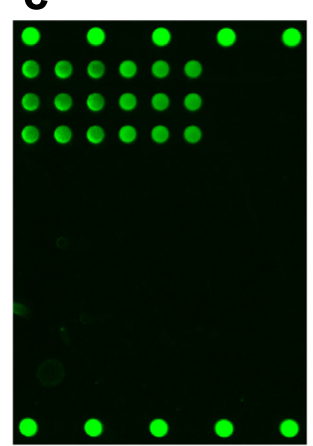

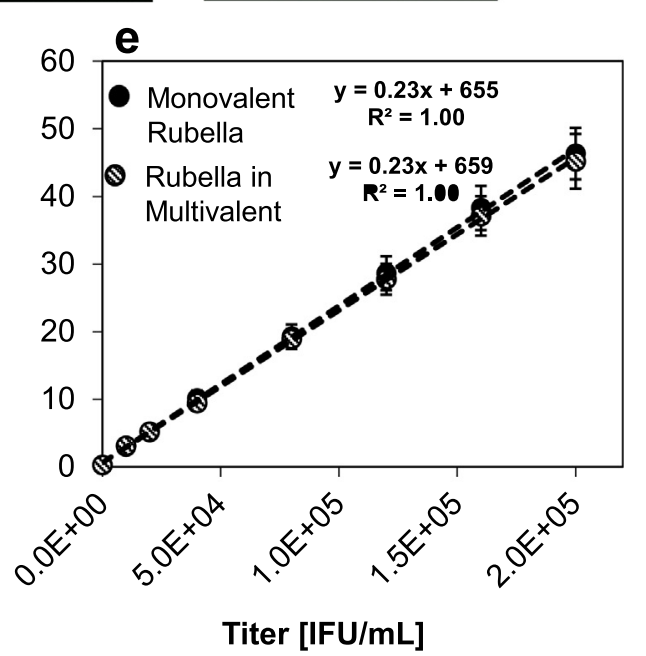

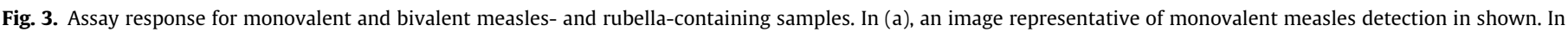

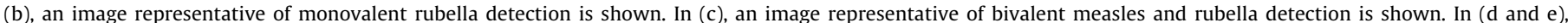

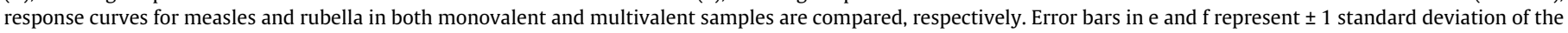
9 replicate antibody spots within the array. 
Measles detection accuracy was unaffected by the presence of any of the tested additives (Fig. 4.a), with all measurements being statistically equivalent to a PBS control. Rubella quantification was statistically unaffected for all matrices except sucrose (Fig. 4.b). When spiked into a solution at $0.38 \%$ sucrose, rubella detection was depressed by $15 \%$ relative to the PBS control. Due to the assay's high precision, this measurement was statistically significantly different than the PBS control ( $\mathrm{p}<0.01$ ), however, was still within a typical $80-100 \%$ accuracy requirement, suggesting the assay produces accurate results in the presence of common MR vaccine additives. In addition, the assay's high sensitivity allows for significant dilution of samples prior to analysis, potentially alleviating the interference by diluting the sucrose to sufficiently low levels.

\subsection{VaxArray MR assay is highly reproducible}

Traditional cell culture assays, such as $\mathrm{CCID}_{50}$, are subject to variability of up to $0.3 \log _{10}$ IFU/mL [19-20]. This translates to relative error of up to $50 \%-100 \%$. These high levels of uncertainty can cause unnecessary wastage and expensive lot rejections.

Bivalent mixtures of measles- and rubella-containing samples were analyzed at low, medium, and high concentrations relative to the LDR. Eight replicates of each bivalent sample were prepared and lysed prior to VaxArray analysis. In accordance with the ICH Guideline for Validation of Analytical Procedures [30], the study was performed by three separate users on three separate days to evaluate intermediate precision.

The assay demonstrated high precision, with $\% \mathrm{CV}<20 \%$ for all tested cases on all days (Fig. 5). The average errors were $6.9 \pm 1.5 \%, 9.9 \pm 6.7 \%$, and $6.5 \pm 3.3 \%$ for users 1,2 , and 3 , respectively, demonstrating no significant differences in the performance of the assay on different days or by different users. In addition, the error across all 72 normalized replicates was $16.2 \%$ for measles and $8.9 \%$ for rubella, demonstrating high intermediate precision and high linearity of dilution. The concentration of the 'low' test sample was $\sim 6 \mathrm{x}$ LLOQ indicating the assay retains high precision even at challenging concentrations. This is a significant improvement over the imprecision often experienced with $\mathrm{CCID}_{50}$, providing a route for same-day, precise measurements for appropriate applications.

\subsection{VaxArray MR assay is sensitive to antigen stability}

MR vaccines are characterized by their infectivity with antigen stability an important consideration for vaccine manufacturers, as conformationally folded protein is one critical driver of infectivity, immune recognition, and vaccine efficacy. Vaccine monovalent bulks containing measles or rubella virus were subjected to $60{ }^{\circ} \mathrm{C}$ for $48 \mathrm{~h}$ to fully denature the viral proteins. Degraded monovalent bulk was combined with intact (non-treated) monovalent bulk at various ratios and analyzed by VaxArray MR to demonstrate the ability of the assay to measure conformationally intact protein. While antigen stability is an important consideration, retention of infectivity is also a critical consideration for live-attenuated vaccines. A second experiment in which CAM-70 measles and RA 27/3 monovalent bulk virus were treated at $+37^{\circ} \mathrm{C}$ for 1 day was also conducted to determine the effect on the VaxArray measurement.

The VaxArray MR assay does not detect denatured proteins, as indicated by complete loss of signal when $+60{ }^{\circ} \mathrm{C}$ heat-treated virus ( $0 \%$ intact (undegraded) sample) was analyzed (Fig. 6 , dashed circles). As the proportion of intact sample was increased (relative to degraded sample), the assay response was linear and indicated slope of 1 and a y-intercept near 0 , confirming detection of only the conformationally folded protein components in a mixture of degraded and intact sample.

When virus was heat-treated at $+37^{\circ} \mathrm{C}$, the sample containing only heat-treated virus did not demonstrate complete signal loss (Fig. 6, filled circles), but rather produced a signal of $\sim 40 \%$ relative to the fully intact sample. As the portion of intact sample was increased (relative to degraded sample), the assay response remained linear, but the slope was $<1$ for each virus, suggesting
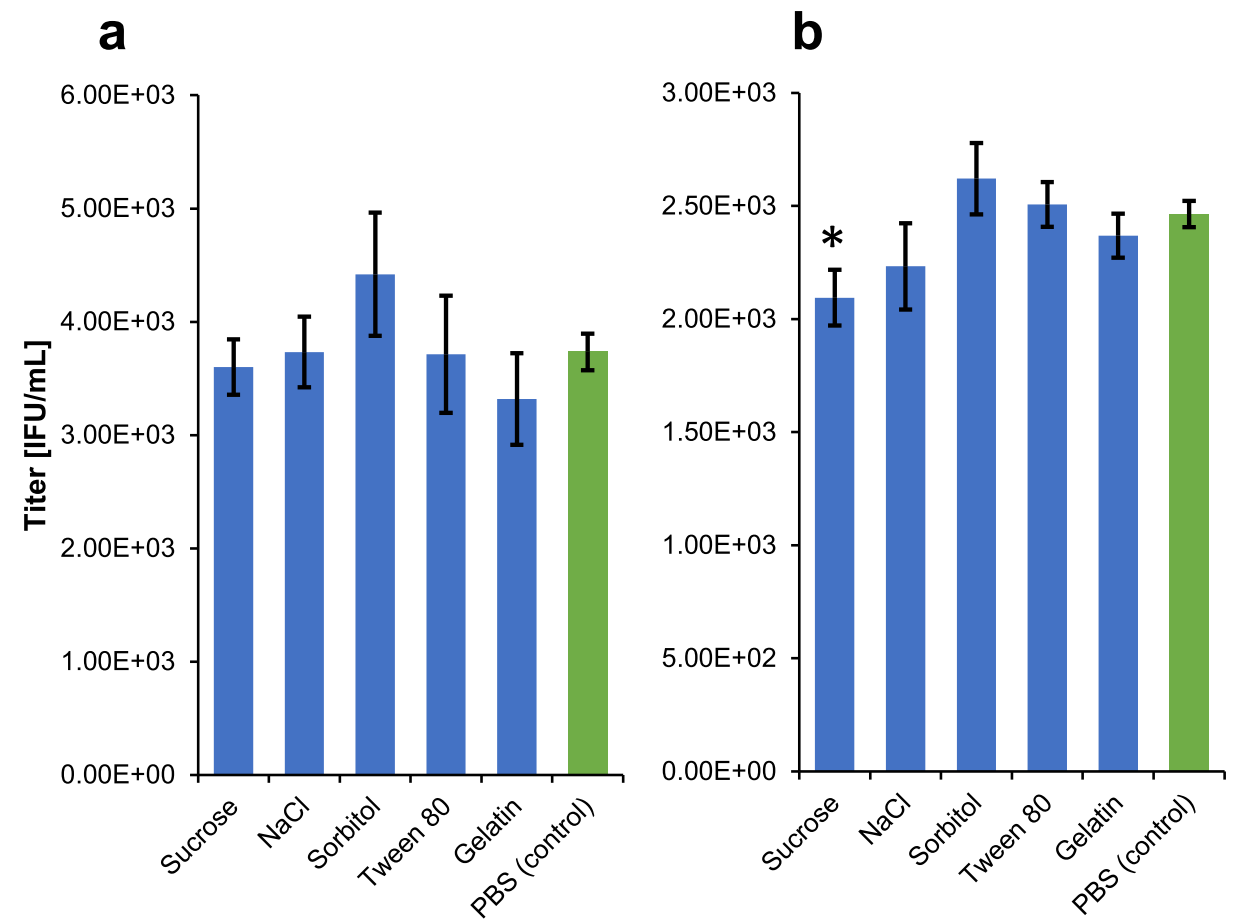

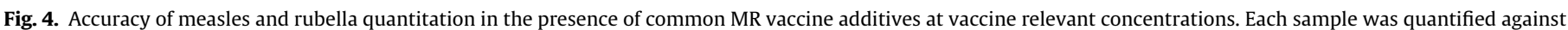

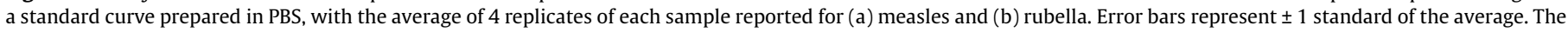
asterisk $\left({ }^{*}\right)$ indicates a statistically significant difference from the PBS control with $\mathrm{p}<0.01$. 

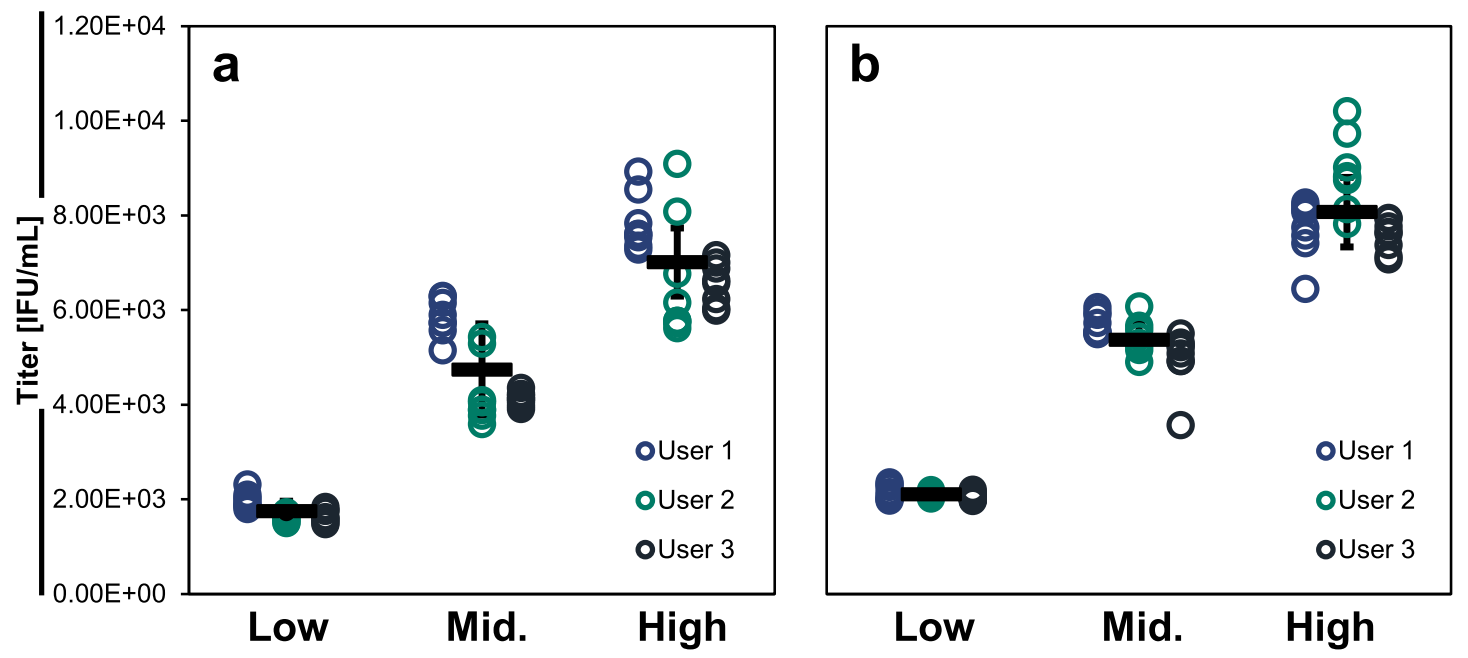

C

$\% C V$ for each mAb

\begin{tabular}{|c|c|c|c|c|}
\hline Conc. & Variations & $\begin{array}{c}\text { Sample Size } \\
(\mathbf{n})\end{array}$ & $\mathbf{M}(\mathbf{i})$ & $\mathbf{R}(\mathbf{i})$ \\
\hline High & Day-to-Day + Analyst-to-Analyst & 24 & 14.5 & 10.4 \\
\hline Mid. & Day-to-Day + Analyst-to-Analyst & 24 & 19.0 & 9.3 \\
\hline Low & Day-to-Day + Analyst-to-Analyst & 24 & 12.0 & 4.9 \\
\hline-- & Day-to-Day + Analyst-to-Analyst + Conc.- \\
to-Conc. & 72 & 16.2 & 8.9 \\
\hline
\end{tabular}

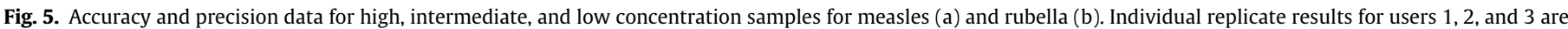

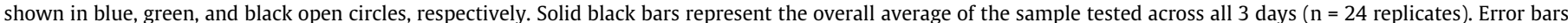

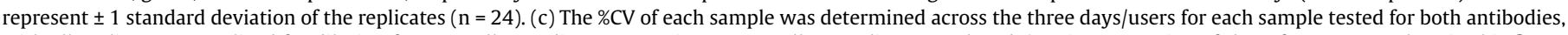

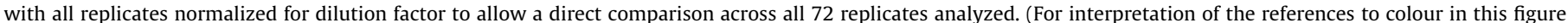
legend, the reader is referred to the web version of this article.)
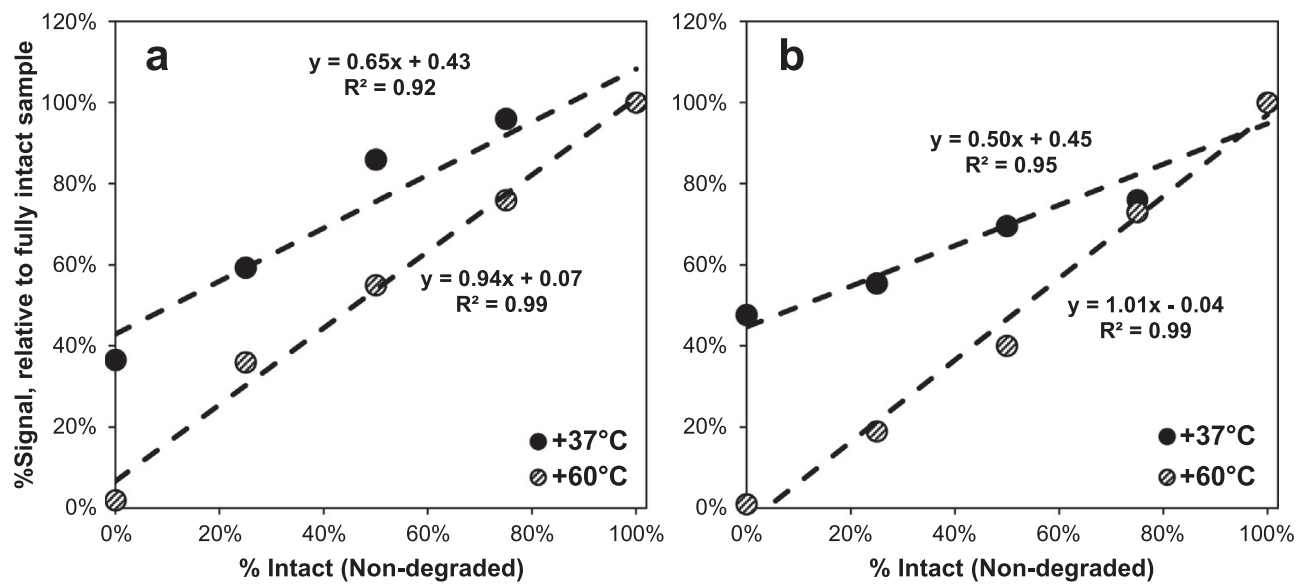

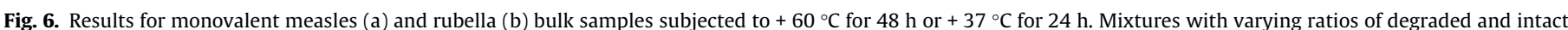

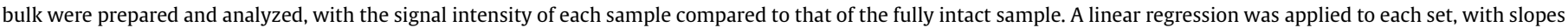
and coefficients of regression $\left(\mathrm{R}^{2}\right)$ as shown.

that exposing measles and rubella viruses to $+37^{\circ} \mathrm{C}$ for 1 day does not fully denature the epitope probed by the antibodies on the microarray. In a separate study (data not shown), the same mono- valent bulk virus samples incubated at $+37^{\circ} \mathrm{C}$ for 1 day and subsequently analyzed byCCID ${ }_{50}$. Both bulks demonstrated complete loss of infectivity, suggesting that protein/antigen stability as mea- 
sured by VaxArray is not directly predictive of infectivity for thermally degraded samples. It is known that the infectivity of measles virus is dependent on the conformational stability of the viral proteins and integrity of the viral membrane [31], and very likely that temperature treatment affects conformation, particularly in these liquid monovalent bulk materials not stabilized with typical final vaccine excipients; however, given that the exact binding epitopes of the antibody probes utilized are not known, we cannot speculate on the underlying mechanism for the assay's difference in response from an infectivity measurement.

\subsection{VaxArray $M R$ measurements are correlated to $C C I D_{50}$ measurements}

To investigate the correlation between VaxArray and $\mathrm{CCID}_{50}$, crude harvest samples for each virus were analyzed by both assays (see Methods section for details).

VaxArray measurements were generally correlated with the $\mathrm{CCID}_{50}$ measurements for both viruses, with positive slopes and $\mathrm{R}^{2}$ of 0.81 and 0.75 for measles and rubella, respectively (Fig. 7). However, the measured concentration is not equivalent to $\mathrm{CCID}_{50}$. This result is not unexpected and is likely because the VaxArray immunoassay measures conformational protein content rather than infectious dose. In the harvest samples analyzed here, there are likely levels of free protein (not associated with intact, infectious viral particles) that vary from batch-to-batch, or within a batch as a function of harvest time or growth condition. These proteins are detected by VaxArray but would not cause CPE in a CCID 50 assay. In addition, if the ratio of free protein to infectious particles is inconsistent for all samples and the calibrant, it is expected that VaxArray measurements will not be identical to CCID $_{50}$ measurements and therefore may be used only as a rapid measure of anti- gen content for these bioprocess samples. We note that the harvest samples utilized in this study were isolated from a single measles lot and 2 different rubella lots, and from numerous different manufacturing conditions within each lot. Based on preliminary studies (data not shown), analysis of samples from a single lot and a single condition (i.e. single standard manufacturing batch) is likely to improve the correlation.

\subsection{VaxArray MR exhibits high accuracy for purified samples}

To evaluate the potential use of the VaxArray MR assay with more purified downstream samples, a set of 2 monovalent measles bulks (CAM-70), and a set of 5 concentrated virus pools (CVP) and 3 monovalent rubella bulks (RA $27 / 3$ strain, from separate manufacturing lots), were analyzed by VaxArray and $\mathrm{CCID}_{50}$. In addition, 3 final vaccines from separate manufacturing lots were reconstituted, and both the measles and rubella components analyzed by VaxArray and $\mathrm{CCID}_{50}$. Fully separate monovalent bulks from unique manufacturing lots with known $\mathrm{CCID}_{50}$ values were used as the VaxArray calibrant.

As summarized in Table 2, the VaxArray measurements for each lot of measles monovalent bulk were only 0.16 and $0.21 \log _{10}$ IFU/ $\mathrm{mL}$ different from the $\mathrm{CCID}_{50}$ measurement, within the typical $\mathrm{CCID}_{50}$ error of $0.30 \log _{10} \mathrm{IFU} / \mathrm{mL}$. The measles component in final vaccines, however, produced VaxArray values that were $0.36,0.60$, and $0.40 \log _{10}$ IFU/mL different from CCID $_{50}$ (Table 2), with VaxArray > infectious dose in all cases. As previously discussed, Fig. 3 indicates that it is unlikely the rubella virus is interfering with the measles measurement, as we observe identical response curves for measles in monovalent and bivalent samples. In addition, Fig. 4 indicates that the measurement accuracy is only minimally affected by the presence of any single vaccine additive. As
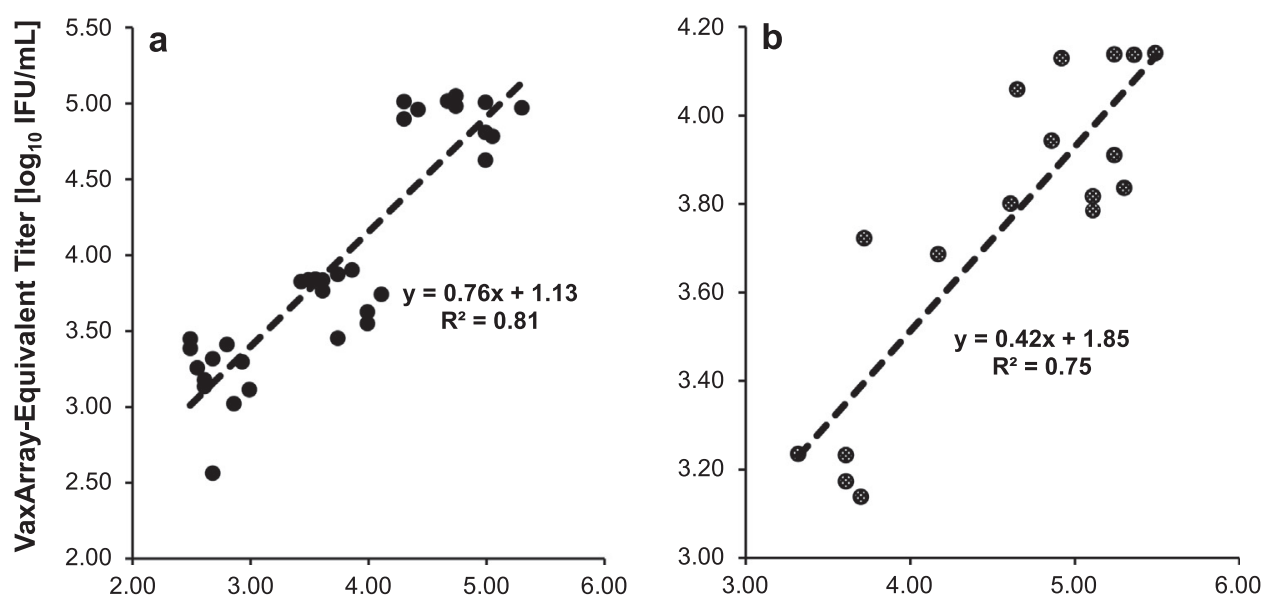

$\mathrm{CCID}_{50}$ Titer $\left[\log _{10} \mathrm{IFU} / \mathrm{mL}\right]$

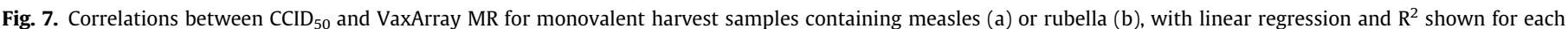
dataset.

Table 2

VaxArray and $\mathrm{CCID}_{50}$ measurements of the measles component of monovalent bulk and final vaccine samples

\begin{tabular}{|c|c|c|c|c|c|}
\hline \multirow{2}{*}{$\begin{array}{l}\text { Sample Type } \\
\text { Manufacturing Lot }\end{array}$} & \multicolumn{2}{|c|}{ Monovalent Bulk } & \multicolumn{3}{|c|}{ Final Vaccine } \\
\hline & A & $\mathrm{B}$ & $\mathrm{C}$ & $\mathrm{D}$ & $\mathrm{E}$ \\
\hline VaxArray Measurement $\left[\log _{10} \mathrm{IFU} / \mathrm{mL}\right]$ & $6.83 \pm 0.06$ & $6.69 \pm 0.05$ & $5.18 \pm 0.13$ & $5.36 \pm 0.08$ & $5.28 \pm 0.08$ \\
\hline $\mathrm{CCID}_{50}$ Measurement $\left[\log _{10} \mathrm{IFU} / \mathrm{mL}\right]$ & 6.99 & 6.90 & 4.82 & 4.76 & 4.88 \\
\hline Difference in Titer $\left[\log _{10}\right.$ IFU/mL] & 0.16 & 0.21 & 0.36 & 0.60 & 0.40 \\
\hline Percent Difference & $2.3 \%$ & $3.0 \%$ & $7.5 \%$ & $12.6 \%$ & $8.1 \%$ \\
\hline
\end{tabular}




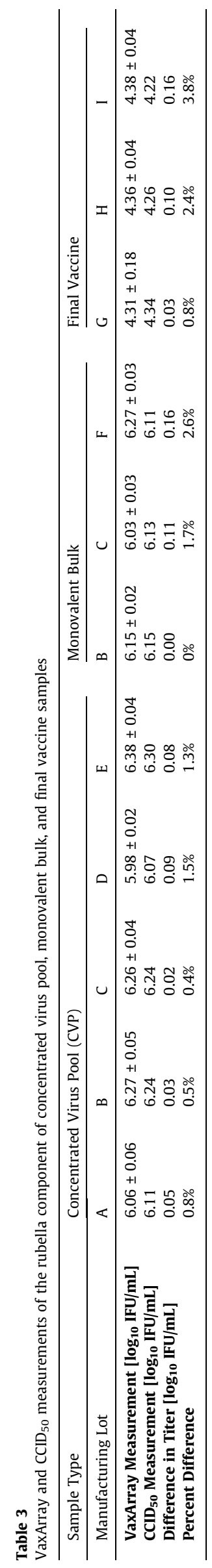

this interference testing was done by spiking single potential interferents into monovalent liquid samples, it is possible that the multiple additives in the lyophilized vaccine affect the measles measurements differently. Therefore, a previously characterized final vaccine sample may be a more appropriate calibrant for the analysis of final vaccine samples. The measles CAM-70 analyzed is also known to exhibit aggregation, so it is possible that the virus is in different states of aggregation in the monovalent bulk as compared to the final vaccine, which may affect VaxArray differently than $\mathrm{CCID}_{50}$.

For rubella, the VaxArray measurements of CVP samples were within $0.05,0.03,0.02,0.09$, and $0.08 \log _{10} \mathrm{IFU} / \mathrm{mL}$, and the rubella monovalent bulks were within $0.16,0.11$, and $0.00 \log _{10}$ IFU/mL of the $\mathrm{CCID}_{50}$ measurements (Table 3 ) for different lots of material. Rubella in final vaccines also showed good agreement with infectious dose, with VaxArray within $0.03,0.10$, and 0.16 $\log _{10} \mathrm{IFU} / \mathrm{mL}$ of $\mathrm{CCID}_{50}$ (Table 3 ), demonstrating high accuracy compared to $\mathrm{CCID}_{50}$ for all three types of samples. In addition, VaxArray demonstrated associated measurement errors between 0.02 and $0.18 \log _{10} \mathrm{IFU} / \mathrm{mL}(\mathrm{n}=2-8$ replicates per sample), significantly lower than the typical $0.30 \log _{10}$ error of the CCID 50 assay.

\section{Conclusion}

While MR vaccines have proven to be extremely efficacious and generate long-lasting immunity, measles and rubella viruses still inflict severe and significant morbidity and mortality, especially in the developing world. More widespread vaccination is critical to eradicate these vaccine-preventable diseases. The current method for standardization of MR vaccine materials, including in-process harvest samples, monovalent bulks, and final vaccines, is the $\mathrm{CCID}_{50}$ assay which is time consuming (1014 days), presents difficulties in the analysis of multivalent samples, and suffers from irreproducibility, creating bottlenecks and costly lot rejections that drive up vaccine cost. New tools are critical to reduce the time-to-result and increase confidence in measurements.

The VaxArray MR assay leverages the proven VaxArray technology to address these hurdles. The assay is sensitive to antigen stability and is generally correlated to infectivity for harvest samples. Quantification of antigens from one virus is unaffected by the presence of antigens from the other virus and most common MR vaccine additives, which, when combined with low limits of quantification (lower than the minimum required for vaccine samples), enable measurement of both antigens in bivalent vaccine samples.

In conjunction with a pre-calibrated internal standard, the VaxArray MR assay demonstrated high accuracy relative to $\mathrm{CCID}_{50}$ for purified samples including concentrated virus pools and monovalent bulks but lower accuracy with crude harvest samples. However, these crude samples spanned multiple manufacturing lots and often different growth conditions within a lot. Further analysis of a larger number of harvest samples from a single lot and single growth condition is warranted to determine if the correlation is improved. Quantification in monovalent bulks using a previously characterized monovalent bulk as the calibrant yields more accurate measurements than in a crude harvest sample, in part, because the matrix is matched. Furthermore, after crude harvest material undergoes purification and concentration steps to arrive at monovalent bulk, the ratio of infectious virions to non-infectious material is likely more consistent. And while VaxArray MR assay results for harvest samples are correlated but not equivalent to $\mathrm{CCID}_{50}$ measurements, when paired with an internal standard previously calibrated by $\mathrm{CCID}_{50}$, the VaxArray MR assay may still provide utility in selecting harvests for 
pooling prior to purification in a fraction of the time relative to $\mathrm{CCID}_{50}$.

In addition to a short time-to-result ( 5 h vs 2 weeks), VaxArray MR exhibited improved reproducibility and precision with overall $\% \mathrm{CV}$ of $15 \%$. While not likely to replace the gold standard infectivity assay used for release testing, this $\sim 10$-fold improvement over CCID $_{50}$ could alleviate a major bottleneck in the characterization and quantification of MR vaccine samples by speeding up early characterization reducing the incidence of costly lot rejections. Further work to develop a sample pre-treatment protocol to improve the correlation with $\mathrm{CCID}_{50}$ harvest samples and for vaccine stability investigations would further increase the utility of the assay throughout the MR vaccine manufacturing process. While the focus of this work is on measles and rubella (MR) vaccines, future addition of mumps and varicella-zoster to the assay would broaden applicability.

Declaration of Interests

K. Rowlen and E. Dawson are InDevR Inc. stockholders. Biological E. Ltd. was provided a VaxArray Imaging System and kits used for analysis at no charge. All other authors are (or were) employed by either InDevR Inc. or Biological E. Ltd. but have no conflicts of interest.

\section{Author Contributions}

Mr. Gillis, Dr. Byrne-Nash, Dr. Dawson, and Ms. Taylor were responsible for experimental design, data interpretation. Mr. Gillis and Dr. Dawson prepared the manuscript; all authors provided edits. Mr. Gillis, Mr. Miller, Ms. Thomas, and Mr. Panchakshari executed experiments. Dr. Rowlen, Dr. Byrne-Nash, and Mr. Gillis are inventors on related intellectual property. Dr. Rowlen and Dr. Dawson provided scientific guidance and program oversight. Dr. Dawson, Dr. Rowlen, and Dr. Senthilkumar Manoharan provided technical support and manuscript review.

\section{Declaration of Competing Interest}

The authors declare that they have no known competing financial interests or personal relationships that could have appeared to influence the work reported in this paper.

\section{Acknowledgements}

This work was supported, in whole or in part, by the Bill \& Melinda Gates Foundation [INV-004629]. Under the grant conditions of the Foundation, a Creative Commons Attribution 4.0 Generic License has already been assigned to the Author Accepted Manuscript version that might arise from this submission. We also thank Batavia Biosciences B.V. for providing measles Schwarz harvest samples for analysis under a material transfer agreement.

\section{References}

[1] World Health Organization. Global Measles and Rubella Strategic Plan: 20122020. 2012.

[2] Measles Signs and Symptoms | CDC n.d. https://www.cdc.gov/measles/ symptoms/signs-symptoms.html (accessed November 11, 2020).

[3] Measles | WHO n.d. https://www.who.int/news-room/fact-sheets/detail/ measles (accessed July 1, 2021).

[4] Measles Complications | CDC n.d. https://www.cdc.gov/measles/ symptoms/complications.html?CDC_AA_refVal=https\%3A\%2F\%2Fwww. cdc.gov\%2Fmeasles\%2Fabout\%2Fcomplications.html (accessed November 11, 2020).

[5] Rubella - Symptoms and causes - Mayo Clinic n.d. https://www.mayoclinic. org/diseases-conditions/rubella/symptoms-causes/syc-20377310 (accessed November 12, 2020).
[6] INTRODUCING RUBELLA VACCINE INTO NATIONAL IMMUNIZATION PROGRAMMES A STEP-BY-STEP GUIDE. 2015.

[7] Congenital Rubella Syndrome Congenital Rubella Syndrome VaccinePreventable Diseases. 2018.

[8] Castillo-Solórzano C, Marsigli C, Bravo-Alcántara P, Flannery B, Ruiz Matus C, Tambini $G$, et al. Elimination of rubella and congenital rubella syndrome in the Americas. J Infect Dis 2011;204(suppl_2):S571-8. https://doi.org/10.1093/ infdis/iir472.

[9] van Boven M, Kretzschmar M, Wallinga J, O’Neill PD, Wichmann O, Hahné S. Estimation of measles vaccine efficacy and critical vaccination coverage in a highly vaccinated population. J R Soc Interf 2010;7(52):1537-44. https://doi. org/10.1098/rsif.2010.0086.

[10] Morse D, O'shea M, Hamilton G, Soltanpoor N, Leece G, Miller E, et al. Outbreak of measles in a teenage school population: the need to immunize susceptible adolescents. Epidemiol Infect 1994;113(2):355-65. https://doi.org/10.1017| S0950268800051785.

[11] Ramsay ME. Measles: the legacy of low vaccine coverage n.d. https://doi.org/ 10.1136/archdischild-2013-304292.

[12] Greaves WL, Orenstein WA, Hinman AR, Nersesian WS. Clinical efficacy of rubella vaccine. Pediatric Infect Dis 1983;2(4):284-6.

[13] Massad E, Azevedo-neto RS, Burattini MN, Zanetta DMT, Coutinho FAB, Yang $\mathrm{HM}$, et al. Assessing the efficacy of a mixed vaccination strategy against rubella in São Paulo, Brazil. Int J Epidemiol 1995;24(4):842-50. https://doi.org 10.1093/ije/24.4.842.

[14] Reef SE, Strebel P, Dabbagh A, Gacic-Dobo M, Cochi S. Progress toward control of rubella and prevention of congenital rubella syndrome - Worldwide, 2009. J Infect Dis 2011;204:S24-7. https://doi.org/10.1093/infdis/jir155.

[15] Hayman B, Pagliusi S. Emerging vaccine manufacturers are innovating for the next decade. Vaccine: X 2020;5:100066. https://doi.org/10.1016/j. jvacx.2020.100066.

[16] Patel MK, Goodson JL, Alexander JP, Kretsinger K, Sodha S v., Steulet C, et al. Progress Toward Regional Measles Elimination - Worldwide, 2000-2019. MMWR Morbidity and Mortality Weekly Report 2020;69:1700-5. https://doi. org/10.15585/mmwr.mm6945a6.

[17] Grigorov B, Rabilloud J, Lawrence P, Gerlier D, Xiang Y. Rapid titration of measles and other viruses: Optimization with determination of replication cycle length. PLoS ONE 2011;6(9):e24135. https://doi.org/10.1371/iournal. pone.0024135.

[18] WHO Technical Report Series, No. 840, Annex 3. 1994.

[19] Pourianfar HR, Javadi A, Grollo L. A colorimetric-based accurate method for the determination of enterovirus 71 titer. Indian J Virol 2012;23(3):303-10. https://doi.org/10.1007/s13337-012-0105-0.

[20] LaBarre DD, Lowy RJ. Improvements in methods for calculating virus titer estimates from TCID50 and plaque assays. J Virol Meth 2001;96(2):107-26. https://doi.org/10.1016/S0166-0934(01)00316-0.

[21] Byrne-Nash RT, Gillis JH, Miller DF, Bueter KM, Kuck LR, Rowlen KL. A neuraminidase potency assay for quantitative assessment of neuraminidase in influenza vaccines. NPJ Vaccines 2019;4(1). https://doi.org/10.1038/s41541019-0099-3.

[22] Byrne-Nash RT, Miller DF, Bueter KM, Gillis JH, Kuck LR, Rowlen KL. VaxArray potency assay for rapid assessment of "pandemic" influenza vaccines. NPJ Vaccines 2018;3:1-11. https://doi.org/10.1038/s41541-018-0080-6.

[23] Kuck LR, Sorensen M, Matthews E, Srivastava I, Cox MMJ, Rowlen KL, et al. Titer on Chip: New Analytical Tool for Influenza Vaccine Potency Determination. PLoS ONE 2014;9(10):e109616. https://doi.org/10.1371/journal. pone.0109616.

[24] Kuck LR, Saye S, Loob S, Roth-Eichhorn S, Byrne-Nash R, Rowlen KL. VaxArray assessment of influenza split vaccine potency and stability. Vaccine 2017;35 (15):1918-25. https://doi.org/10.1016/i.vaccine.2017.02.028.

[25] Spearman C. Review of the Method of "Right and Wrong Cases" ('Constant Stimuli') without Gauss's Formula. Psychol Bull 1909;6:27-8. https://doi.org/ 10.1037/h0063767.

[26] Kärber G. Beitrag zur kollektiven Behandlung pharmakologischer Reihenversuche. Naunyn-Schmiedebergs Archiv Für Experimentelle Pathologie Und Pharmakologie 1931;162(4):480-3. https://doi.org/10.1007/ BF01863914.

[27] Knipe DM, Howley JM. Field's Virology. sixth ed. Philadelphia: Lippincott, Williams, \& Wilkins; 2013.

[28] Chaye HH, Mauracher CA, Tingle AJ, Gillam S. Cellular and Humoral Immune Responses to Rubella Virus Structural Proteins E1, E2, and C. J Clin Microbiol 1992;30(9):2323-9.

[29] FDA, CBER. Package Insert - Measles, Mumps, and Rubella Virus Vaccine Live. n.d.

[30] ICH HARMONISED TRIPARTITE GUIDELINE VALIDATION OF ANALYTICAL PROCEDURES: TEXT AND METHODOLOGY O2(R1) Guideline on Validation of Analytical Procedures: Methodology developed to complement the Parent Guideline 2005.

[31] Kissmann J, Ausar SF, Rudolph A, Braun C, Cape SP, Sievers RE, et al. Stabilization of measles virus for vaccine formulation. Human Vaccines 2008;4(5):350-9. 